\title{
CSF concentrations of 5-methyltetrahydrofolate in a cohort of young children with autism
}

John Shoffner, MD

Barbara Trommer, MD

Audrey Thurm, PhD

Cristan Farmer, PhD

William A. Langley III,

$\mathrm{PhD}$

Laura Soskey, MA

Aldeboran N. Rodriguez, MD

Precilla D'Souza, PNP

Sarah J. Spence, MD, $\mathrm{PhD}$

Keith Hyland, PhD

Susan E. Swedo, MD

Correspondence to Dr. Swedo:

swedos@mail.nih.gov
Supplemental data at Neurology.org

\section{ABSTRACT}

Objective: To examine the association between cerebral folate deficiency and autism, this study examined CSF 5-methyltetrahydrofolate (5-MTHF) concentrations in a group of young children with autism, investigated the natural variation in CSF 5-MTHF over time, and assessed the relationship between CSF 5-MTHF and symptoms.

Methods: CSF was collected from 67 children with a diagnosis of DSM-IV-TR autistic disorder (age, mean \pm SD $43 \pm 11$ months), with a second CSF sample obtained 1-3 years later on 31 of these subjects (time to follow-up, $30 \pm 8$ months).

Results: At time 1, 7\% (5/67) of participants had 5-MTHF <40 nmol/L. At follow-up, 23\% (7/31) of participants had $5-\mathrm{MTHF}<40 \mathrm{nmol} / \mathrm{L}$ (only one of whom had been low at time 1 ). A moderate correlation with a very wide confidence interval (CI) was observed between time 1 and time 2 CSF 5-MTHF measurements (Pearson $r[p]=0.38$ [0.04]; 95\% Cl 0.02-0.64). Neither the CSF 5-MTHF levels nor changes over time correlated with the clinical features of autism.

Conclusions: CSF 5-MTHF levels vary significantly over time in an unpredictable fashion and do not show a significant relationship to typical clinical features of autism. Reduced CSF 5-MTHF levels are a nonspecific finding in autism. Our data do not support the use of lumbar puncture for assessment of CSF 5-MTHF in autism. Neurology ${ }^{\circledR}$ 2016;86:2258-2263

\section{GLOSSARY}

5-HIAA = 5-hydroxyindolacetic acid; 5-MTHF = 5-methyltetrahydrofolate; ADOS = Autism Diagnostic Observation Schedule; $\mathbf{A S D}=$ autism spectrum disorder; CFD = cerebral folate deficiency; $\mathbf{C l}=$ confidence interval; DSM-IV-TR = Diagnostic and Statistical Manual of Mental Disorders, 4th edition, Text Revision; FBP = folate transport protein binding antibodies; HVA = homovanillic acid; IgG = immunoglobulin G; LP = lumbar puncture.

Cerebral folate deficiency (CFD) is defined broadly as any neurologic syndrome associated with an abnormally low CSF concentration of 5-methyltetrahydrofolate (5-MTHF) in the presence of normal peripheral folate. ${ }^{1-4}$ There is no agreed-upon threshold for low CSF 5-MTHF; most studies establish cutoffs based on the study controls, ${ }^{4}$ with most converging upon values around $40 \mathrm{nmol} / \mathrm{L}$. Clinical features associated with CFD include global developmental delays, ataxia, and myoclonic seizures, and it may be associated with increased homocysteine ${ }^{5}$ and neurotransmitter abnormalities. ${ }^{6,7}$ In rare cases, CFD results from a FOLR1 genetic mutation, and idiopathic CFD is found in some patients with mitochondrial disorders (e.g., Kearns-Sayre) and other neurodegenerative syndromes including Rett syndrome. ${ }^{8-13}$ Three case series report on CFD in autism spectrum disorder (ASD), each concluding that there may be some causal relationship. ${ }^{14-16}$ One study reported some evidence of neurologic improvement associated with CSF 5-MTHF normalization. ${ }^{16}$

While there is speculation that dysfunctional folate metabolism might play a role in the pathogenesis of autism, ${ }^{17,18}$ these hypotheses are limited by the lack of pediatric CSF controls. Further, there is no systematic documentation of whether low CSF 5-MTHF in ASD is accompanied by the neurologic symptoms of CFD. We obtained repeat CSF 5-MTHF samples from young children with autism participating in a longitudinal phenotyping study, who were

From Medical Neurogenetics (J.S., W.A.L., K.H.); Georgia State University (J.S.), Atlanta; Pediatrics \& Developmental Neuroscience Branch (B.T., A.T., C.F., L.S., A.N.R., P.D., S.J.S., S.E.S.), National Institute of Mental Health, National Institutes of Health, Bethesda, MD; State University of New York Downstate Medical Center (B.T.), Brooklyn; and Department of Neurology (S.J.S.), Boston Children's Hospital, MA. Go to Neurology.org for full disclosures. Funding information and disclosures deemed relevant by the authors, if any, are provided at the end of the article. 
not recruited based on neurologic symptoms of CFD. Our objectives were to characterize their CSF 5-MTHF levels, determine variability in CSF 5-MTHF over time, and evaluate the relationship between CSF 5-MTHF levels and symptoms of autism. As folate receptor autoantibodies have been reported to be present in a significant number of children with ASD, ${ }^{19}$ anti-folate transport protein binding antibodies (immunoglobulin G [IgG] antiFBP) were also assessed.

METHODS Participants. Participants were drawn from a longitudinal study of 106 children with autism aged 2-6 years at baseline; 67 of these participants underwent at least one lumbar puncture (LP) during the study period of 2006-2013 and were included in the current report. A subset of these children $(\mathrm{n}=31)$ had a second LP. Eligible participants met DSM-IV-TR criteria for autistic disorder on the basis of the Autism Diagnosis Interview-Revised (or a Toddler version) ${ }^{20}$ and the Autism Diagnostic Observation Schedule (ADOS). ${ }^{21}$ Exclusion criteria included neurologic conditions such as cerebral palsy or aggressive or maladaptive behaviors that precluded participation in behavioral testing. Demographic data are shown in table 1.

Standard protocol approvals, registrations, and patient consents. All procedures were approved by an NIH Institutional Review Board. Written informed consent was obtained from the guardians of each participant. This study is registered with ClinicalTrials.gov (NCT00298246).

Clinical assessment. Information about birthweight, gestational age, neonatal care, history of epilepsy, use of medications, and use of special diets or dietary supplements was obtained from medical records and parents/guardians. Adaptive behavior was assessed with the Vineland Adaptive Behavior Scale, Second Edition. ${ }^{22}$ Cognitive functioning was assessed with either Mullen Scales of Early Learning ${ }^{23}$ or the Differential Ability Scales, Second Edition. ${ }^{24}$ Developmental quotient (ratio of age equivalents to chronological age) was calculated for nonverbal cognitive ability, due to the confounding effects of autism symptoms on the verbal subscale. Severity of autism symptoms was rated with the ADOS calibrated severity score, which indexes the child's score by age and verbal ability $(1=$ no symptoms, $10=$ most severe $){ }^{25}$

Procedures. Participants adhered to their usual regimens in the days leading up to the testing, but all were required to fast for at least 6 hours prior to sedation for the LP (MRI and venipuncture were also performed). Blood samples were collected 1-2 hours prior to the LP, except in one child from whom the serum folate sample was collected 10 months after the LP. Per study protocol, LPs were performed between approximately $10 \mathrm{AM}$ and $11 \mathrm{AM}$. The study protocol included an LP for a portion of participants in the autism group; however, 39 participants were not eligible due to predefined age criteria or parental refusal, resulting in 67 time 1 LPs. Thirty-one (46\%) of the 67 participants had a repeat LP 1-3 years (mean \pm SD $29.6 \pm 8.3$ months) after their initial evaluation; these participants were not selected by the investigators for any clinical indication. The remainder of the sample either declined $(n=12)$ or were not eligible due to changes in study procedures $(n=24)$. Time 1 and time 2 are used to refer to data from the first and second LPs, respectively.

CSF samples were processed within 20 minutes of collection by centrifuging for 10 minutes at $1,000 \mathrm{RPM}$ at $4^{\circ} \mathrm{C}$, and then flash frozen in 10 and $20 \mu \mathrm{L}$ aliquots and stored at $-80^{\circ} \mathrm{C}$. Blood was collected in SST tubes and sent to the NIH Clinical Center laboratory where serum total folate was measured by the NIH Clinical Center laboratory using competitive immunoassay method. Analyses of CSF 5-MTHF and the neurotransmitter metabolites 5-hydroxyindolacetic acid (5-HIAA), homovanillic acid (HVA), and 3-hydroxymethyldopa were performed by Medical Neurogenetics (Atlanta, GA) (http://www.mnglab.com/). CSF 5-MTHF was measured using high-performance liquid chromatography with electrochemical and fluorescence detection, as previously described. ${ }^{26}$ Medical Neurogenetics also performed analysis of autoantibodies binding to the human folate receptor in the serum (IgG anti-FBP) using an ELISA-based assay. Plasma and CSF amino acids were measured by Mayo Medical Labs (Rochester, MN) using quantitative analysis by liquid chromatography-tandem mass spectrometry. CSF analysis was performed with batched samples upon completion of sample ascertainment. Thus, results and referrals for potential intervention to participants with low CSF 5-MTHF occurred after the second sample collection.

Although typically developing controls were recruited for the broader natural history study, ethical constraints prevented LPs in these participants. Thus, comparison data for CSF 5-MTHF were obtained from Medical Neurogenetics, representing 540 samples

Table 1 Participant demographic and phenotypic information

\begin{tabular}{|c|c|c|c|c|c|c|}
\hline & \multicolumn{3}{|l|}{ Visit 1} & \multicolumn{3}{|l|}{ Visit 2} \\
\hline Age, mo, mean \pm SD & $42.23 \pm 11.81$ & $44.82 \pm 8.35$ & $42.66 \pm 11.31$ & $74.13 \pm 15.23$ & $65.25 \pm 7.96$ & $71.84 \pm 14.16$ \\
\hline Age range, mo & $24-83$ & $30-58$ & $24-83$ & $42-116$ & $54-77$ & $42-116$ \\
\hline NVDQ range & $31.56-103.63$ & $33.78-74.73$ & $31.56-103.63$ & $21.05-115.34$ & $34.27-73.58$ & 21.05-115.34 \\
\hline ADOS CSS, mean $\pm S D$ & $7.71 \pm 1.6$ & $7.64 \pm 1.12$ & $7.7 \pm 1.53$ & $6.65 \pm 2.17$ & $7.75 \pm 1.04$ & $6.94 \pm 1.98$ \\
\hline CSS range & $4-10$ & $6-9$ & $4-10$ & $2-10$ & $6-9$ & $2-10$ \\
\hline
\end{tabular}

Abbreviations: 5-MTHF = 5-methyltetrahydrofolate; ADOS = Autism Diagnostic Observation Schedule; CSS = calibrated severity score. 
Figure Distribution of CSF 5-methyltetrahydrofolate (5-MTHF) values at time 1 and time 2

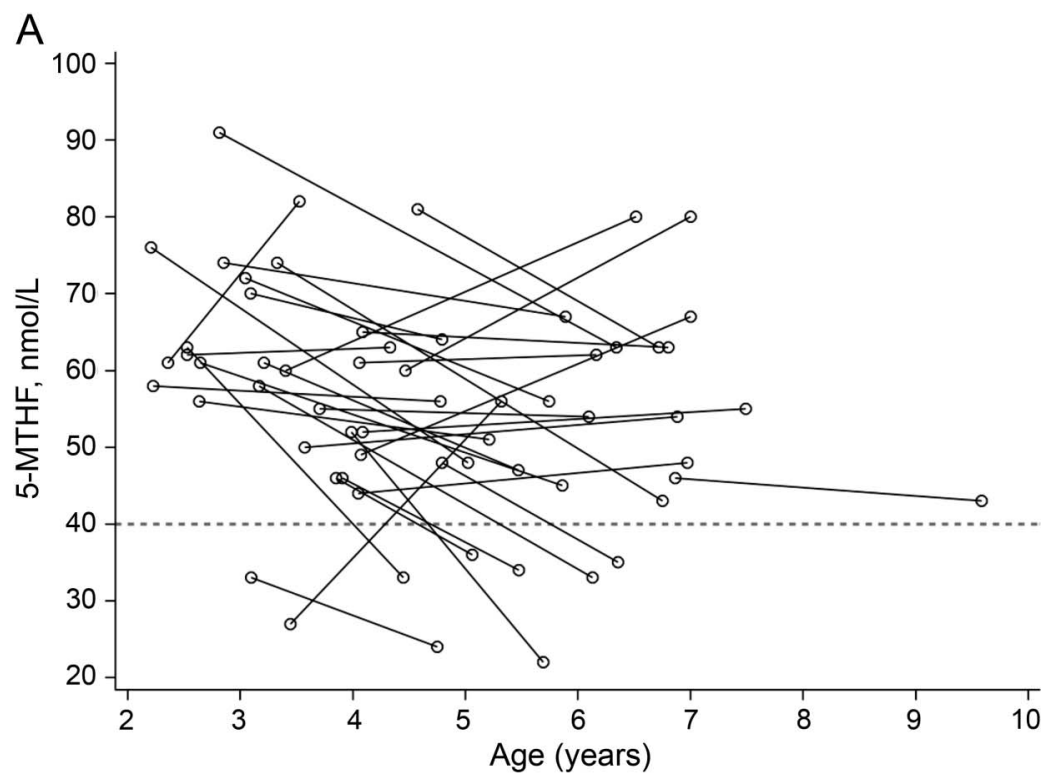

B

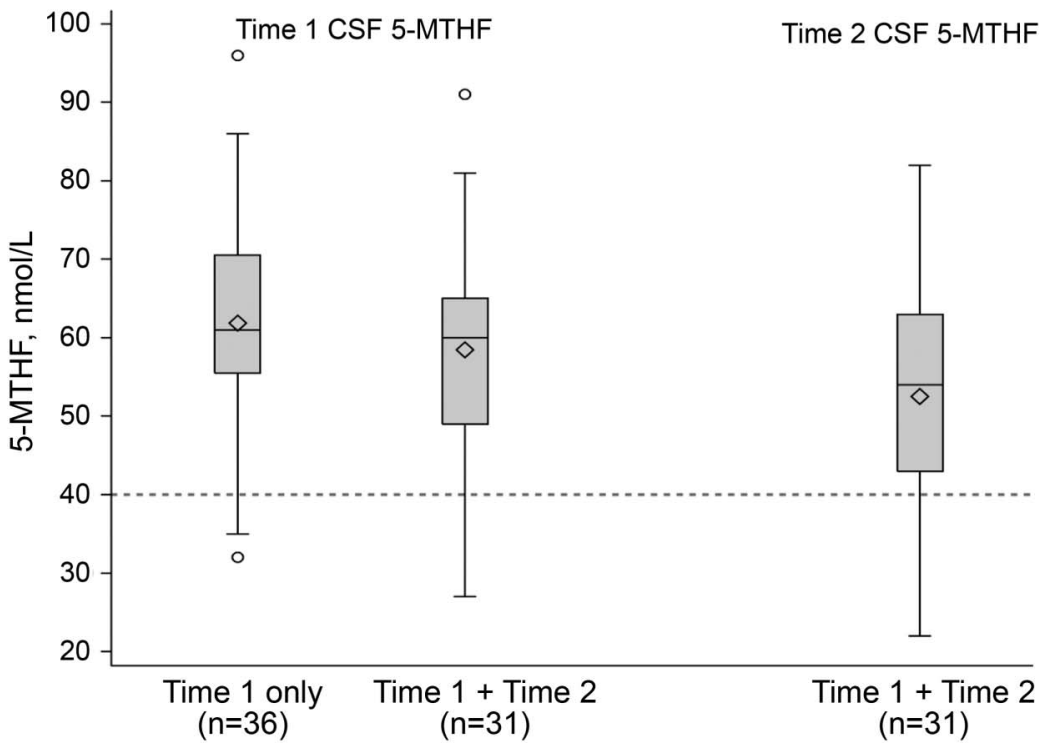

Mean age at visit 1 was $42.7 \pm 11.3$ months. Mean time to follow-up was $29.7 \pm 8.3$ months (range 14-43 months). Medical Neurogenetics provided CSF 5-MTHF reference ranges: $40-$ $150 \mathrm{nmol} / \mathrm{L}$ for $2-5$ years, $40-128 \mathrm{nmol} / \mathrm{L}$ for $5-10$ years. In the current study, values $<40$ $\mathrm{nmol} / \mathrm{L}$ were considered low. (A) Individual CSF 5-MTHF values; (B) mean values at each time point. Accounting for age, mean CSF 5-MTHF did not change significantly between time 1 and time $2(F=0.16, p=0.69)$.

sent for CSF 5-MTHF analysis based on neurologic disorder symptom presentation between 1995 and 2000. The dataset included individuals with a wide range of neurologic disorders, but excluded patients with infections, seizures, movement disorders, inborn errors of folate, $B_{12}$, or serine, and those receiving anticonvulsants, methotrexate, or other drugs known to interfere with folate metabolism. In the current study, only participants within the age range of the ASD sample were used for comparison $(\mathrm{n}=140)$.

Statistical analysis. The general linear model was the primary method of analysis in this study. Within-group change over time was assessed using repeated measures, using fixed effects for visit and age. Group differences were evaluated controlling for age. The $\chi^{2}$ test was used to determine the statistical significance of comparisons on categorical variables; Fisher exact test was reported where cell sizes were small. All statistical procedures were carried out using SAS version 9.3. ${ }^{27}$ The $\alpha$ was set to 0.05 .

RESULTS In the 67 children with autism, CSF 5-MTHF at baseline ranged from 27.0 to 96.0 $\mathrm{nmol} / \mathrm{L}$ (figure, table 2). Based on a cutoff of 40 $\mathrm{nmol} / \mathrm{L}, 5(7 \%)$ children were classified as low CSF 5-MTHF. Serum folate was normal in all participants (table 2), and none had evidence of megaloblastic anemia or other signs of peripheral folate deficiency. A correlation between CSF 5-MTHF and serum folate was not observed (Pearson $r=0.004$, $p=0.97$.

A second CSF sample was available for $31(46 \%)$ participants (figure). Mean time to follow-up was $29.7 \pm 8.3$ months (range 14-43 months). A moderate correlation with a very wide confidence interval (CI) was observed between time 1 and time 2 CSF 5-MTHF measurements $(\mathrm{n}=31$; Pearson $r=0.38$, $p=0.04 ; 95 \%$ CI 0.02-0.64). CSF 5-MTHF decreased in $21(68 \%)$ participants. Although the group average decrease was only $-6 \%$ (time 1 mean \pm SD $58.5 \pm 13.5 \mathrm{nmol} / \mathrm{L}$; time 2 mean \pm SD $52.5 \pm 15.5 \mathrm{nmol} / \mathrm{L}$ ), significant variation of CSF 5-MTHF was observed within individual participants. Further, when age was controlled, this decrease was not statistically significant $(F=0.16$, $p=0.69)$. It is noteworthy that $7(23 \%)$ participants had CSF 5-MTHF values less than $40 \mathrm{nmol} / \mathrm{L}$ at time 2 , though only one of these children had been classified as low at time 1. Of the 5 participants classified as low at time 1, 2 had follow-up (both received multivitamins but no folic acid supplementation), and only one remained low at time 2 .

Time 1 samples were compared to those from an age-matched comparison group obtained from Medical Neurogenetics $(n=140$; mean \pm SD $3.9 \pm 1.3$ years). In the comparison sample, values ranged from 20.5 to $151.2 \mathrm{nmol} / \mathrm{L}($ mean $\pm \mathrm{SD} 77.5 \pm 26.2$ nmol/L). Twelve (9\%) participants had low 5-MTHF, with values ranging from $20.5 \mathrm{nmol} / \mathrm{L}$ to $39.1 \mathrm{nmol} / \mathrm{L}$ (mean $\pm \mathrm{SD} 30.5 \pm 6.6 \mathrm{nmol} / \mathrm{L}$ ). Age was initially entered into the analysis of covariance model, but was excluded as it was nonsignificant. Mean CSF 5-MTHF values were significantly lower in the children with autism than in the reference group $\left(F_{1,205}=25.67, p<0.0001\right.$; Cohen $d=$ $0.8,95 \%$ CI $0.5-1.1)$.

CSF folate receptor protein IgG anti-FBP. At time 1, 4 (6\%) participants had elevated antibodies binding to the folate receptor protein (IgG anti-FBP) (66, 71,84 , and $153 \mathrm{pmol} / \mathrm{mL}$ ). All of these participants 
Table 2 Laboratory results $(n=67)$

$\begin{array}{llll} & \text { Mean } \pm \text { SD } & \text { Range } & \text { IQR } \\ \text { CSF 5-MTHF, nmol/L } & 60.3 \pm 13.6 & 27-96 & 52-70 \\ \text { Serum folate, nmol/L } & 73.9 \pm 27.5 & 29-208 & 50-94 \\ \text { CSF:serum ratio } & 0.9 \pm 0.4 & 0.2-1.8 & 0.6-1.1\end{array}$

Abbreviations: 5-MTHF = 5-methyltetrahydrofolate; IQR = interquartile range. All serum folate samples were collected on the same day as each patient's CSF sample with the exception of one child whose serum folate sample was collected 10 months after CSF collection.

had CSF 5-MTHF above $40 \mathrm{nmol} / \mathrm{L}$ (49, 52, 61, and $66 \mathrm{nmol} / \mathrm{L})$. One of the participants with elevated antibodies at time 1 had a second LP, at which time no IgG anti-FBP were detected. However, at time 2, 4 (13\%) different participants had elevated IgG anti-FBP (77, 110, 159, and $226 \mathrm{pmol} / \mathrm{mL}$; corresponding 5-MTHF 56, 63, 43, and $56 \mathrm{nmol} / \mathrm{L}$ ).

Other laboratory values. Serum homocysteine levels were normal in all samples. No participants exhibited abnormalities in CSF 3-O-methyl DOPA. At time 1, 3 children had low 5-HIAA, and at time 2, 5 different children had low 5-HIAA. One of the 5 children with low 5-HIAA at time 2 also had low HVA. None of these children with low neurotransmitter levels had CSF 5 -MTHF $<40 \mathrm{nmol} / \mathrm{L}$ at either time point. No CSF or plasma amino acid abnormalities coincided with values of $<40 \mathrm{nmol} / \mathrm{L}$ CSF 5-MTHF.

Clinical correlates. We explored possible clinical correlates of 5-MTHF status using separate general linear models, controlling for age (table 3). CSF 5-MTHF was not significantly associated with any measures of adaptive behavior, cognitive ability, or ASD symptom severity. Nor was change in CSF 5-MTHF level associated with change in any of the adaptive behavior, cognitive, or autism symptom measures.

Overall, the clinical evaluation revealed few abnormalities for any of the children, including the 11 who had 5 -MTHF $<40 \mathrm{nmol} / \mathrm{L}$ at either time point. Of 5 children with a seizure disorder, one had CSF 5 MTHF $<40 \mathrm{nmol} / \mathrm{L}$ at time 2 only (medications included oxcarbazepine, clonidine, and multivitamin). Physical and neurologic examinations conducted at time $1(n=63)$ were unremarkable except for neurobehavioral abnormalities and hypotonia in 19 of 57 children, 2 of whom had CSF 5 -MTHF $<40 \mathrm{nmol} / \mathrm{L}$ (data on hypotonia were missing for 6 children who received physical examinations). The percentage of children on special diets is indicated in table e-1 on the Neurology ${ }^{\circledR}$ Web site at Neurology.org. At a given timepoint, participants with CSF 5-MTHF less than $40 \mathrm{nmol} / \mathrm{L}$ were likelier to consume a gluten-free or casein-free diet than the remainder of the sample (time $1,60 \%$ vs $35 \%$, nonsignificant; time $2,86 \%$ vs $13 \%, p<0.01)$. At both time 1 and time 2, no participant with low CSF 5-MTHF was taking valproic acid, which may cause folate depletion.

Genetic studies of children in the sample revealed 3 pathogenic abnormalities. Specifically, chromosomal microarray analysis found a pathogenic copy number variation in one child with $2 \mathrm{p} 25.3$ duplication and 9p24.3-p23 deletion translocation confirmed with FISH, clinically indicated targeting sequencing found one child with a pathogenic $15 q 14$ mutation (i.e., Legius syndrome), and karyotyping had identified one child with Turner syndrome. Only the child with Turner syndrome had CSF 5-MTHF $<40 \mathrm{nmol} / \mathrm{L}$, observed only at time 2 .

DISCUSSION The primary finding of this study is that the clinical features of autism were not associated with absolute levels of CSF 5-MTHF or with the considerable variability in CSF 5-MTHF over time. CSF 5-MTHF less than $40 \mathrm{nmol} / \mathrm{L}$ was observed in 11 of 67 children in 1 of $2 \mathrm{LPs}$; of the 5 participants with low 5-MTHF at time 2, only one had been low at time 1. Importantly, low 5-MTHF levels were not associated with motor or other neurologic symptoms previously reported among patients with CFD. With the exception of diet, individuals with low 5-MTHF levels in our study group were indistinguishable from other children with ASD in both behavior and laboratory assays. Further, variability in CSF 5-MTHF concentrations occurred across timepoints, with low CSF 5-MTHF values found at time 1 but not time 2 , or vice versa.

All studies that assess pediatric CSF are limited by the absence of reference values from healthy children as well as the lack of understanding of the natural variability over time of this metabolite. Our results emphasize that the natural variation on CSF 5MTHF can be large, calling into question the value of single timepoint measurements. Since ethical restrictions preclude LPs from being performed for research studies among healthy, typically developing children, results for the children with autism were compared against a group of children with neurologic problems, including CFD. CSF 5-MTHF values ranged up to $151.2 \mathrm{nmol} / \mathrm{L}$ in this comparison group, while the maximum value observed in the autism group at time 1 was only $96.0 \mathrm{nmol} / \mathrm{L}$. The lower bounds of the samples were similar, as were the rates of values less than $40 \mathrm{nmol} / \mathrm{L}$ (autism, 7\%; comparison, 9\%). It is worth noting that the comparison data were obtained between 1995 and 2000, so they were not collected or analyzed contemporaneously with the ASD data. The current ASD data are more similar in range to another recent study of ASD, ${ }^{19}$ and to one in children without ASD (analyzed by a different 
Table 3 No significant relationships between CSF 5-MTHF and phenotypic variables

\begin{tabular}{|c|c|c|c|}
\hline & B (SE) & $t$ & p Value \\
\hline \multicolumn{4}{|l|}{ Predicting time 1 CSF 5-MTHF } \\
\hline \multicolumn{4}{|l|}{ Developmental quotients } \\
\hline Full-scale & $0.07(0.15)$ & 0.46 & 0.65 \\
\hline Nonverbal & $-0.02(0.13)$ & -0.15 & 0.89 \\
\hline Verbal & $0.15(0.18)$ & 0.83 & 0.41 \\
\hline \multicolumn{4}{|l|}{ Vineland standard scores } \\
\hline Communication & $0.01(0.11)$ & 0.09 & 0.93 \\
\hline Daily living skills & $0.01(0.08)$ & 0.14 & 0.89 \\
\hline Motor skills & $0.06(0.07)$ & 0.84 & 0.40 \\
\hline Socialization & $-0.09(0.06)$ & -1.51 & 0.14 \\
\hline Adaptive behavior composite & $-0.004(0.06)$ & -0.07 & 0.95 \\
\hline ADOS calibrated severity score & $-0.01(0.02)$ & -0.39 & 0.70 \\
\hline \multicolumn{4}{|l|}{ Predicting change in CSF 5-MTHF } \\
\hline \multicolumn{4}{|l|}{ Change in developmental quotients } \\
\hline Full-scale & $0.003(0.18)$ & 0.02 & 0.98 \\
\hline Nonverbal & $-0.01(0.2)$ & -0.05 & 0.96 \\
\hline Verbal & $0.04(0.18)$ & 0.21 & 0.84 \\
\hline \multicolumn{4}{|l|}{ Change in Vineland standard scores } \\
\hline Communication & $0.13(0.18)$ & 0.75 & 0.46 \\
\hline Daily living skills & $0.13(0.15)$ & 0.89 & 0.38 \\
\hline Motor skills & $-0.57(0.24)$ & -2.36 & 0.03 \\
\hline Socialization & $0.12(0.13)$ & 0.87 & 0.39 \\
\hline Adaptive behavior composite & $0.14(0.12)$ & 1.17 & 0.25 \\
\hline ADOS calibrated severity score & $0.002(0.02)$ & 0.07 & 0.95 \\
\hline
\end{tabular}

Abbreviations: 5-MTHF = 5-methyltetrahydrofolate; ADOS = Autism Diagnostic Observation Schedule; B = unstandardized regression coefficient; SE = standard error of B.

Vineland scores are standard scores (mean $=100, S D=15)$. Seven children were out of age range for Vineland Adaptive Behavior Scale Motor Skills at time 2. "Predicting time 1 CSF 5-MTHF" section provides statistical relationships between time 1 CSF 5-MTHF and time 1 phenotypic scores, controlling for age. "Predicting change in CSF 5-MTHF" section provides statistical relationships between changes in both CSF 5-MTHF and phenotypic scores from time 1 to time 2, controlling for age and time to follow-up.

laboratory), ${ }^{28}$ with the exception of values lower than $40 \mathrm{nmol} / \mathrm{L}$. Given the constraints on LPs in healthy children, future studies may consider control groups comprised of children who receive LPs for specific medical problems such as headaches with no pleocytosis or idiopathic intracranial hypertension.

Existing studies generally converge upon 40 $\mathrm{nmol} / \mathrm{L}$ as the lower limit of normal CSF 5MTHF. ${ }^{2,3}$ Using the limit of $40 \mathrm{nmol} / \mathrm{L}, 11$ children with autism (but without other neurologic abnormalities) were found to have low CSF 5-MTHF concentrations at either time 1 or time 2 . The lack of stability across measurements and the failure to find correlation between behavioral measures and CSF 5-MTHF levels are evidence against the role of CSF 5-MTHF in the etiology of autism. In fact,
CSF 5-MTHF decreased over time for a subgroup of children with autism, consistent with cross-sectional studies showing decreasing CSF 5-MTHF with age in healthy children. ${ }^{28}$ This gives rise to the possibility that detection of a low CSF 5-MTHF at a single time point merely identifies natural biological variability, rather than a causal mechanism of autism. This may also be true of the other disorders that have sometimes been linked to CFD. Furthermore, only 4 participants had elevated levels of anti-folate binding receptor protein antibodies at either time point, and none had low CSF 5-MTHF. This suggests that autoantibodies were not a major contributor to low CSF 5-MTHF concentrations in this cohort and that the relationship between autism and autoantibodies may only be present in certain patient groups. ${ }^{29}$ The results of this study do not permit determination of the cause for CSF 5-MTHF variability. 5-MTHF levels did not appear to impact ASD symptoms in this sample, nor were decreasing 5-MTHF concentrations associated with worsening symptoms.

This investigation represents the largest collection of CSF from an ASD cohort to date. It is also unique for having longitudinal samples and analyses. Low CSF 5-MTHF levels were found in only a small fraction of children with ASD, and were neither consistent across time nor associated with clinical symptoms or behavioral characteristics. Our data do not support the use of LP for assessment of CSF 5-MTHF in autism.

Our results demonstrate that all studies investigating CSF 5-MTHF must consider the natural variation of CSF 5-MTHF over time. The results of this study underscore the need for pediatric CSF normative data, which has been a persistent limitation in the study of CNS activity in children with neurologic disease.

\section{AUTHOR CONTRIBUTIONS}

John Shoffner: contributed to design of the study, interpretation of data, and revising the manuscript. Barbara Trommer: contributed to analysis and interpretation of the data and drafting the manuscript. Audrey Thurm: contributed to conceptualization and design of the study, analysis and interpretation of data, and drafting and revising the manuscript. Cristan Farmer: contributed to analysis and interpretation of data and drafting and revising the manuscript. William Langley: contributed to analysis of the data. Laura Soskey: contributed to analysis of the data. Aldeboran N. Rodriguez: contributed to analysis of the data. Precilla D'Souza: contributed to analysis of the data. Sarah Spence: contributed to the conceptualization of the study. Keith Hyland: contributed to design of the study, interpretation of data, and revising the manuscript. Susan Swedo: contributed to conceptualization and design of the study, interpretation of data, and revising the manuscript. Dr. Swedo is the Guarantor of this research.

\section{STUDY FUNDING}

Supported by the Intramural Program of the National Institute of Mental Health of the National Institutes of Health, NCT00298246, 06-M-0102, and ZIA MH002914. John Shoffner, MD, William A. Langley, III, PhD, and Keith Hyland, PhD, were supported by the 
Department of Defense under award numbers W81XWH-09-1-0367 and W81XWH-10-1-0547. Views and opinions of and endorsements by the author(s) do not reflect those of the US Army, the Department of Defense, NIMH, NIH, HHS, or the United States Government.

\section{DISCLOSURE}

J. Shoffner holds stock and/or stock options in Medical Neurogenetics. B. Trommer, A. Thurm, C. Farmer, W. Langley III, L. Soskey, A. Rodriguez, P. D'Souza, and S. Spence report no disclosures relevant to the manuscript. K. Hyland is co-owner of Medical Neurogenetics. S. Swedo reports no disclosures relevant to the manuscript. Go to Neurology.org for full disclosures.

Received October 21, 2015. Accepted in final form March 14, 2016.

\section{REFERENCES}

1. Djukic A. Folate-responsive neurologic diseases. Pediatr Neurol 2007;37:387-397.

2. Hyland K, Shoffner J, Heales SJ. Cerebral folate deficiency. J Inherit Metab Dis 2010;33:563-570.

3. Ramaekers VT, Blau N. Cerebral folate deficiency. Dev Med Child Neurol 2004;46:843-851.

4. Pérez-Duenas B, Ormazábal A, Toma C, et al. Cerebral folate deficiency syndromes in childhood: clinical, analytical, and etiologic aspects. Arch Neurol 2011;68:615-621.

5. Ho PI, Ashline D, Dhitavat $S$, et al. Folate deprivation induces neurodegeneration: roles of oxidative stress and increased homocysteine. Neurobiol Dis 2003;14:32-42.

6. Hyland K. Clinical utility of monoamine neurotransmitter metabolite analysis in cerebrospinal fluid. Clin Chem 2008;54:633-641.

7. Serrano M, Garcia-Silva MT, Martin-Hernandez E, et al. Kearns-Sayre syndrome: cerebral folate deficiency, MRI findings and new cerebrospinal fluid biochemical features. Mitochondrion 2010;10:429-432.

8. Allen RJ, DiMauro S, Coulter DL, Papadimitriou A, Rothenberg SP. Kearns-Sayre syndrome with reduced plasma and cerebrospinal fluid folate. Ann Neurology 1983;13:679-682.

9. Garcia-Cazorla A, Quadros EV, Nascimento A, et al. Mitochondrial diseases associated with cerebral folate deficiency. Neurology 2008;70:1360-1362.

10. Ramaekers VT, Hansen SI, Holm J, et al. Reduced folate transport to the CNS in female Rett patients. Neurology 2003;61:506-515.

11. Blau N, Bonafe L, Krageloh-Mann I, et al. Cerebrospinal fluid pterins and folates in Aicardi-Goutières syndrome: a new phenotype. Neurology 2003;61:642-647.

12. Ramaekers VT, Rothenberg SP, Sequeira JM, et al. Autoantibodies to folate receptors in the cerebral folate deficiency syndrome. N Engl J Med 2005;352:1985-1991.

13. Neul JL, Maricich SM, Islam M, et al. Spinal fluid 5-methyltetrahydrofolate levels are normal in Rett syndrome. Neurology 2005;64:2151-2152.

14. Moretti P, Peters SU, Del Gaudio D, et al. Brief report: autistic symptoms, developmental regression, mental retardation, epilepsy, and dyskinesias in CNS folate deficiency. J Autism Dev Disord 2008;38:1170-1177.

15. Mangold S, Blau N, Opladen T, et al. Cerebral folate deficiency: a neurometabolic syndrome? Mol Genet Metab 2011;104:369-372.

16. Ramaekers VT, Blau N, Sequeira JM, Nassogne MC, Quadros EV. Folate receptor autoimmunity and cerebral folate deficiency in low-functioning autism with neurological deficits. Neuropediatrics 2007;38:276-281.

17. Main PA, Angley MT, Thomas P, O’Doherty CE, Fenech M. Folate and methionine metabolism in autism: a systematic review. Am J Clin Nutr 2010;91:1598-1620.

18. Rinaldo P, Hahn MD. Inborn errors of amino acid, organic acid and fatty acid metabolism. In: Ca Burtis EA, Bruns DE, ed. Tiezt Textbook of Clinical Chemistry and Molecular Diagnosis, 4th ed. St. Louis: WB Saunders; 2005:2207-2247.

19. Frye RE, Sequeira JM, Quadros EV, James SJ, Rossignol DA. Cerebral folate receptor autoantibodies in autism spectrum disorder. Mol Psychiatry 2013;18: 369-381.

20. Lord C, Rutter M, Le Couteur A. Autism Diagnostic Interview-Revised: a revised version of a diagnostic interview for caregivers of individuals with possible pervasive developmental disorders. J Autism Dev Disord 1994;24: 659-685.

21. Lord C, Risi S, Lambrecht L, et al. The Autism Diagnostic Observation Schedule-Generic: a standard measure of social and communication deficits associated with the spectrum of autism. J Autism Dev Disord 2000;30: 205-223.

22. Sparrow SS, Cicchetti DV, Balla DA. Vineland Adaptive Behavior Scales, 2nd ed. Circle Pines, MN: AGS Publishing; 2005.

23. Mullen EM. Mullen Scales of Early Learning. Circle Pines, MN: American Guidance Service; 1995.

24. Elliott CD. Differential Ability Scales, 2nd ed. San Antonio: The Psychological Corporation; 2007.

25. Gotham K, Pickles A, Lord C. Standardizing ADOS scores for a measure of severity in autism spectrum disorders. J Autism Dev Disord 2009;39:693-705.

26. Surtees R, Hyland K. Cerebrospinal fluid concentrations of $\mathrm{S}$-adenosylmethionine, methionine, and 5-methyltetrahydrofolate in a reference population: cerebrospinal fluid S-adenosylmethionine declines with age in humans. Biochem Med Metab Biol 1990;44:192-199.

27. SAS [computer program]. Version 9.3. Cary, NC: SAS Institute; 2012.

28. Ormazabal A, García-Cazorla A, Pérez-Dueñas B, et al. Determination of 5-methyltetrahydrofolate in cerebrospinal fluid of paediatric patients: reference values for a paediatric population. Clin Chim Acta 2006;371:159-162.

29. Sequeira JM, Ramaekers VT, Quadros EV. The diagnostic utility of folate receptor autoantibodies in blood. Clin Chem Lab Med 2013;51:545-554. 